\title{
OPEN Preparation of Novel Mesoporous Silica Using a Self-Assembled Graphene Oxide Template
}

\begin{abstract}
Kyeong-Won Park ${ }^{1}$ \& Oh-Yun Kwon ${ }^{2}$
Novel mesoporous silicas rolled with silica sheets with 2D regular spacing were prepared using a self-assembled graphene oxide (GO) template formed by mixing GO with Pluronic123 (P123). Selfassembled GO templated mesoporous silicas (SGT-PMS) showed well-developed X-ray diffraction peaks with $d$-spacings of $9.8-10.8 \mathrm{~nm}$ depending on the amount of $\mathrm{GO}$, indicating mesoporous structures. The specific surface areas increased from 603.8 to $861.2 \mathrm{~m}^{2} \mathrm{~g}^{-1}$ on adding GO. The pore size distribution was in the range $5.1-5.8 \mathrm{~nm}$ and pore volume in the range $0.80-0.99 \mathrm{~m}^{3} \mathrm{~g}^{-1}$. The SEM images of SGT-PMS showed irregular elliptical particles with various sizes. TEM images showed that the cross section of SGT-PMS particles comprises a roll of silica sheets with $2 \mathrm{D}$ regular spacing. The pore walls of SGT-PMS are firmer and thicker than those for PMS without $G O$ as indicated by the corresponding intensities of $Q^{3}$ and $\mathrm{Q}^{4}$ signals. These results were explained well by the self-assembled $\mathrm{GO}$ templating mechanism.
\end{abstract}

Periodic mesoporous silica (PMS) has attracted great interest in fields such as separation, adsorption, catalysis, optics, and biomedicine due to excellent properties such as high porosities, large specific surface areas, low densities, tunable and large pore sizes, surface hydrophilicity, and biocompatibility ${ }^{1-10}$.

Zeolites, which are microporous materials with three-dimensional aluminosilicate skeleton structures and constant pore sizes of $\leq 1 \mathrm{~nm}$, are also referred to as molecular sieves ${ }^{11,12}$.

They were also expected to be suitable catalyst carriers for the catalytic cracking process of the heavy oil. However, they had to improve the limited pore size for practical use. To overcome this limitation, researchers in Mobil synthesized MCM- $41^{13}$ and MCM- $48^{14}$, which drastically increased studies on the synthesis and application of PMS in nanochemical fields as adsorbents and catalyst carriers ${ }^{14-19}$.

PMS related materials such as MCM- $41^{13}$, SBA- $15^{1}$, and FSM- $16^{20,21}$ are typically synthesized using a surfactant micelle-template. Their pore characteristics were also altered by changing the structure and morphology of micelles, which was achieved by adjusting the surfactant type, molecular structure, concentration, and additives ${ }^{17,19,22,23}$.

In particular, MCM-41 related materials synthesized using a cationic surfactant micelle-template were expected to be highly suitable as catalyst carriers because of their regular and uniform pore structure. However, their pore structures collapsed easily under hydrothermal conditions or at temperatures higher than $700{ }^{\circ} \mathrm{C}$ because of their thin pore walls. SBA-15 has also attracted attention as an adsorbent and catalyst carrier in nanochemical fields because it is thermally stable and has excellent pore characteristics like large surface areas and regular pore sizes of $\sim 5 \mathrm{~nm}^{1}$. We also reported that PMS with stable pore structure could be prepared using a nonionic surfactant micelle-template ${ }^{23}$. Dipole interactions occur between the ethylene oxide chain in the nonionic surfactant and metal hydroxide ions ${ }^{18,23-27}$. When the metal hydroxide ions are stabilized and solidified by these interactions, the pore walls of the PMS are expected to be thicker than the ethylene oxide chain length. The formation of a thick pore wall results in a large specific surface area and an excellent thermal stability.

In recent years, the synthesis of PMS using graphene oxide $(\mathrm{GO})$ as a template ${ }^{28,29}$ and graphene-PMS hybrids such as sandwich-type nanocomposites of $\mathrm{GO}$ and $\mathrm{PMS}^{30-32}$ have attracted interest. The extraordinary properties of graphene such as single atomic layer carbon structure, excellent electrical conductivity, mechanical stiffness, thermal conductivity, and chemical reactivity ${ }^{33-38}$ have been combined with other functionalities by forming nanocomposites with metal and metal oxides ${ }^{28,39-42}$.

${ }^{1}$ Department of Chemistry and Faculty of General Education, Gyeongsang National University, Jinju, 52828, Republic of Korea. ${ }^{2}$ Department of Chemical and Biomolecular Engineering, Chonnam National University, Yosu, Chonnam, 59626, Republic of Korea. *email: nano2k@nate.com; oykwon@jnu.ac.kr 


\begin{tabular}{|l|l|l|l|}
\hline Sample & GO $(\mathbf{m g})$ & $\mathbf{P 1 2 3}(\mathrm{g})$ & TEOS $(\mathrm{g})$ \\
\hline SGT & 3 & 1 & 0 \\
\hline PMS-0 & 0 & 1 & 2.15 \\
\hline SGT-PMS-1.5 & 1.5 & 1 & 2.15 \\
\hline SGT-PMS-2.2 & 2.2 & 1 & 2.15 \\
\hline SGT-PMS-3.0 & 3 & 1 & 2.15 \\
\hline
\end{tabular}

Table 1. Mass ratios of the reactant solutions for the preparation of SGT, PMS-0 and SGT-PMS-x.

In this study, novel PMS rolled with 2D silica sheets are successfully synthesized using a self-assembled GO template (SGT). When GO sheets are added to a Pluronic123 (P123) solution, hydrophilic groups of P123 molecules are adsorbed on the surface of GO sheets by interacting with $\pi$-electrons of GO sheet, resulting in more hydrophobic GO sheets. Such hydrophobic GO sheets form self-assembled GO aggregates, which form a roll stacked with GO sheets due to the neutral interaction between surface alkyl groups. Tetraethyl orthosilicate (TEOS) is easily introduced into the hydrophobic galleries between GO sheets thereby composing self-assembled GO aggregates. The gelation of TEOS in the gallery produces rolls sandwiched with silica sheets between GO sheets. Their calcination results in a roll of two-dimensional silica sheets with very regular spacing.

\section{Materials and methods}

Preparation of GO. GO was prepared according to the modified Hummer's method ${ }^{29,43,44}$. Briefly, in a 2-L three-necked round-bottom flask, commercial graphite powder (Aldrich, $5 \mathrm{~g}$ ) and $\mathrm{NaNO}_{3}(3.75 \mathrm{~g})$ were added to concentrated $\mathrm{H}_{2} \mathrm{SO}_{4}(350 \mathrm{~mL})$. This mixture was stirred in an ice-water bath, and $20 \mathrm{~g}$ of $\mathrm{KMnO}_{4}$ was slowly added over $1 \mathrm{~h}$, followed by continuous stirring for $2 \mathrm{~h}$ in the ice-water bath. After the mixture was stirred vigorously for 2 days at room temperature, $700 \mathrm{~mL}$ of a $5 \mathrm{wt} . \% \mathrm{H}_{2} \mathrm{SO}_{4}$ aqueous solution was added over $1 \mathrm{~h}$ with stirring at a constant temperature of $98^{\circ} \mathrm{C}$. The resultant mixture was further stirred for $2 \mathrm{~h}$ at $98^{\circ} \mathrm{C}$. After the temperature was reduced to $60^{\circ} \mathrm{C}, 15 \mathrm{~mL}$ of $\mathrm{H}_{2} \mathrm{O}_{2}(30 \mathrm{wt}$. $\%$ aqueous solution) was added, and the mixture was stirred for $2 \mathrm{~h}$ at room temperature. To remove extraneous oxidation products and other inorganic impurities, the resultant mixture was purified by repeating the following procedure 20 times: centrifugation, removal of the supernatant liquid, dispersion of the solid using vigorous stirring, and ultrasonication for $1 \mathrm{~h}$ at a power of $150 \mathrm{~W}$. The resultant solid was recovered by centrifugation, washed with deionized water and ethanol $(\mathrm{EtOH}) \mathrm{until}^{+}$was removed, and then dried in air at $40^{\circ} \mathrm{C}$.

Synthesis of SGT-PMS-x. The weight ratios of the reactant solutions are listed in Table 1. Briefly, $1.0 \mathrm{~g}$ of P123 $\left(M_{\mathrm{av}}=5800\right.$, Aldrich) was dissolved in a mixture of $30 \mathrm{~mL}$ of $2-\mathrm{N} \mathrm{HCl}$ and $8.0 \mathrm{~mL}$ of distilled water at $38^{\circ} \mathrm{C}$. Then a certain amount of as-prepared GO (weighing $0,1.5,2.2$ or $3.0 \mathrm{mg}$ ) was added and homogeneously dispersed under sonication. For each solution, $2.15 \mathrm{~g}$ of TEOS (Aldrich) was added with vigorous stirring for $5 \mathrm{~min}$, resulting in gel type precipitation. Here, SGT was also prepared by homogeneously dispersing under sonication after adding $3.0 \mathrm{mg}$ of as-prepared GO to $30 \mathrm{ml}$ of above P123 solution without TEOS. The resultant solutions were aged for $5 \mathrm{~h}$ at $80^{\circ} \mathrm{C}$. As-synthesized solid samples were recovered by filtration and washed with deionized water, and then dried for $3 \mathrm{~h}$ at $80^{\circ} \mathrm{C}$. The dried samples were labelled as SGT and as-synthesized SGT-PMS- $\mathrm{x}(\mathrm{x}=1.5,2.2,3.0 \mathrm{mg})$ and $\mathrm{x}$ represented the different weights $(\mathrm{mg})$ of GO added. Finally, dried powders of as-synthesized SGT-PMS- $\mathrm{x}$ were calcined in a furnace for $5 \mathrm{~h}$ at $600^{\circ} \mathrm{C}$ in air, which removed the $\mathrm{P} 123$ and GO templates. The final samples were also labelled as SGT-PMS- $\mathrm{x}(\mathrm{x}=1.5,2.2,3.0)$ and $\mathrm{x}$ represented different weights (mg) of GO added. For comparison, the ordered mesoporous silica prepared without GO was labelled as PMS-0.

Characterization. The powder X-ray diffraction (XRD) patterns of the samples were recorded on a Bruker D8-Advance X-ray powder diffractometer using $\mathrm{Cu} K \alpha$ radiation $(\lambda=0.1542 \mathrm{~nm})$ with scattering angles $(2 \theta)$ of $1^{\circ}-10^{\circ}$, operating at $40 \mathrm{keV}$, with a cathode current of $20 \mathrm{~mA}$. Fourier transform infrared (FT-IR) were recorded using Attenuated Total Reflectance (ATR) sampling accessory on the Nicolet iS50 FT-IR spectrometer. X-ray photoelectron spectra (XPS) were recorded using a VersaProbe, Ulvac-PHI, with Al Ka excitation radiation $(\mathrm{hv}=1486.6 \mathrm{eV})$. The pressure in the analyser was maintained at approximately $6.7 \times 10^{-7} \mathrm{~Pa}$. XPS data was processed using a DS 300 data system.

Scanning electron micrographs were obtained using a JEOL JSM-840A scanning electron microscope (SEM). The transmission electron micrographs (TEM) were obtained with a JEOL JEM-200 CX transmission electron microscope operated at $200 \mathrm{kV}$, using a thin-section technique. The powder samples were embedded in epoxy resin and then sectioned with ultra-microtome. Atomic force microscopy (AFM) images were obtained using an AutoProbe CP/MT scanning probe microscope (XE-100(PSIA)). Imaging was performed in non-contact mode using a V-shaped Ultralever probe B (Park Scientific Instruments, B-doped Si with a frequency $f_{\mathrm{c}}=78.6 \mathrm{kHz}$, spring constant $k=2.0-3.8 \mathrm{~N} \mathrm{~m}^{-1}$, and nominal tip radius $=10 \mathrm{~nm}$ ). All images were collected under ambient conditions at $50 \%$ relative humidity and $23^{\circ} \mathrm{C}$ with a scanning raster rate of $1 \mathrm{~Hz}$. Samples for AFM were prepared by depositing dispersions of GO in EtOH on a freshly cleaved mica surface (Ted Pella Inc. Prod No. 50) and allowing them to air-dry.

Solid-state ${ }^{29}$ Si MAS NMR spectra was recorded on a Bruker CXP-100 spectrometer at a resonance frequency of $19.89 \mathrm{MHz}$ with a $45^{\circ}$ pulse and a recycle delay of $7 \mathrm{~s}$. Raman spectra were obtained using a Jobin Yvon/ Horiba LabRAM spectrometer equipped with an integral microscope (Olympus BX 41). A 514.5-nm Ar-laser was used as an excitation source. Samples were sonicated in EtOH and three drops were placed on a glass slide 

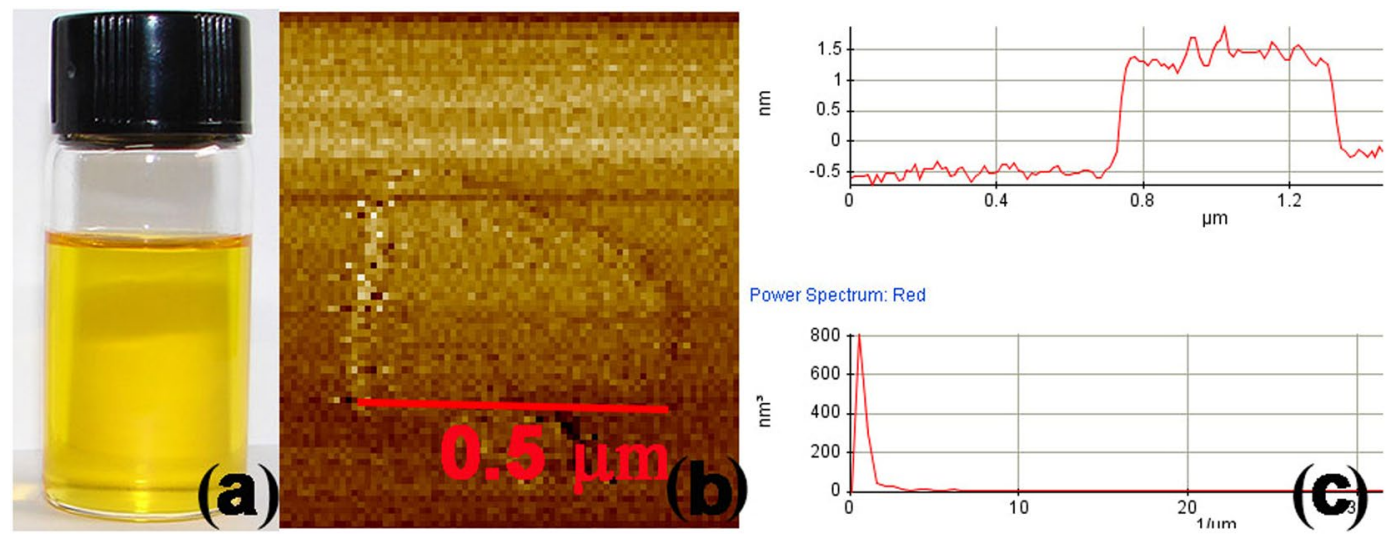

Power Spectrum: Red

Figure 1. (a) Photograph of the GO solution, (b) AFM image of a GO sheet, (c) topography and height profile of a GO sheet.

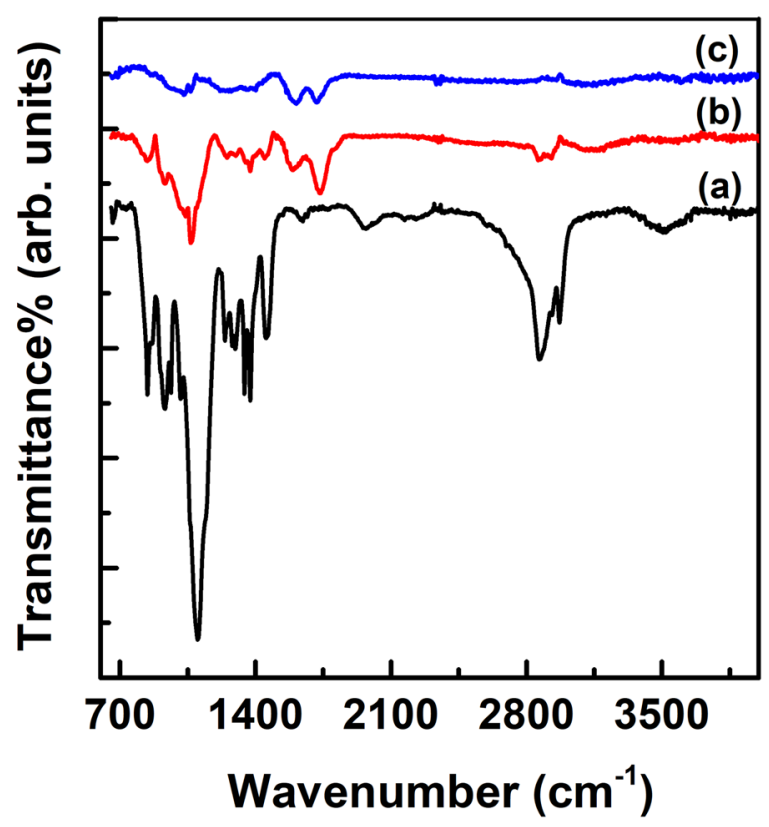

Figure 2. FT-IR spectra of (a) P123 (b) GO, and (c) SGT.

for observation. The samples were viewed using green and red laser apparatuses with maximum magnifications of $50 \times$ and $100 \times$, respectively. $\mathrm{N}_{2}$ adsorption isotherms were obtained at $77 \mathrm{~K}$ using a nitrogen sorption instrument (Micromeritics ASAP 2020). Pore size distributions were calculated by the Barrett-Joyner-Halenda (BJH) method using the adsorption branches of the isotherms.

\section{Results and discussion}

Figure 1(a) shows a photograph of the GO solution, showing well-formed GO as indicated by the yellow colour ${ }^{29}$; Fig. 1(b,c) show AFM images indicating GO particles with a thickness of $\sim 1 \mathrm{~nm}$ and length of $\sim 0.5 \mu \mathrm{m}$, corresponding with previous reports $s^{45,46}$. However, the GO prepared in this work comprises single or multiple sheets, because AFM images can be discriminated well for the single GO sheets.

Figure 2 shows FT-IR peaks for the P123, GO and SGT. P123 peaks [Fig. 2(a)] arises at $800 \sim 1200 \mathrm{~cm}^{-1}(-\mathrm{C}-$ C-), 2850 2900 $\mathrm{cm}^{-1}(-\mathrm{C}-\mathrm{H})$ and $3200 \sim 3600 \mathrm{~cm}^{-1}(-\mathrm{O}-\mathrm{H})$ and GO peaks [Fig. 2(b)] at $800 \sim 1200 \mathrm{~cm}^{-1}(-\mathrm{C}-$ $\mathrm{C}-), 1750 \mathrm{~cm}^{-1}(-\mathrm{C}=\mathrm{C}-)$. Whereas, the characteristic peaks of $\mathrm{P} 123$ and GO largely decrease or nearly disappear in SGT peaks [Fig. 2(c)], indicating that P123 interacts with GO. When GO sheets are added to a Pluronic123 (P123) solution, hydrophilic groups of P123 molecules are adsorbed on the surface of GO sheets by interacting with $\pi$-electrons of GO sheet, resulting in more hydrophobic GO sheets. Such hydrophobic GO sheets form self-assembled GO aggregates, which form a roll stacked with GO sheets owing to the neutral interaction between the surface alkyl groups. In particular, the absence of $-\mathrm{OH}$ peak in SGT indicates that $\mathrm{OH}$ groups of $\mathrm{P} 123$ interact with $\pi$-electrons of GO sheet. 


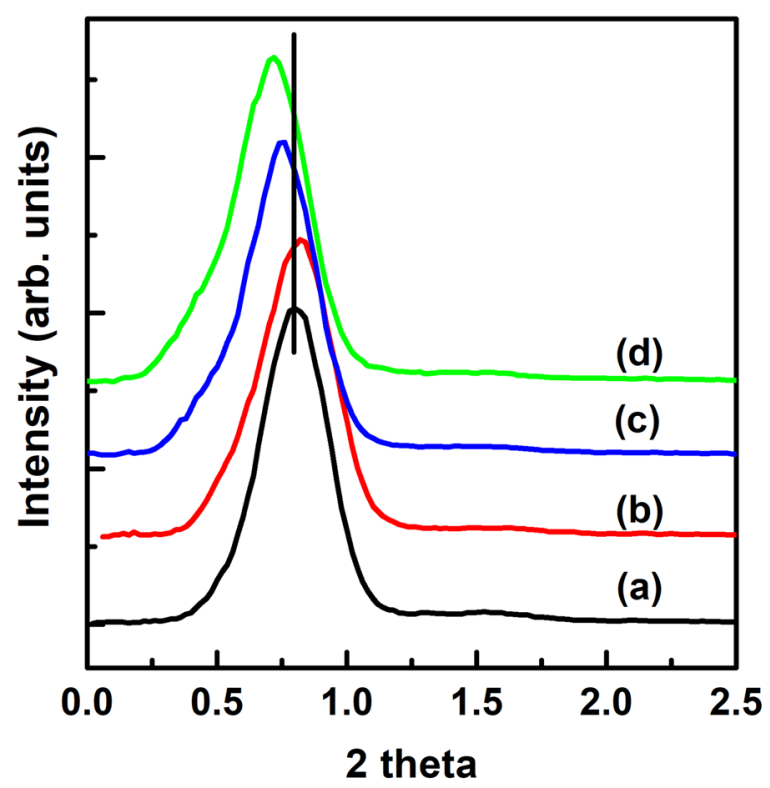

Figure 3. XRD patterns for as-synthesized samples of (a) PMS-0, (b) SGT-PMS-1.5, (c) SGT-PMS-2.2, and (d) SGT-PMS-3.0.

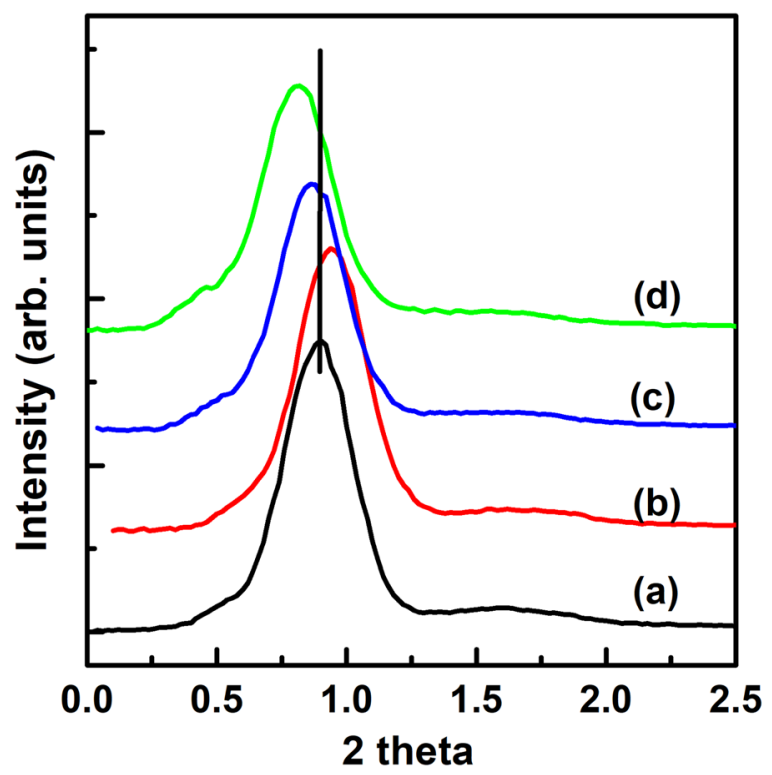

Figure 4. XRD patterns of (a) PMS-0, (b) SGT-PMS-1.5, (c) SGT-PMS-2.2, and (d) SGT-PMS-3.0.

Figure 3 shows the XRD peaks for as-synthesized PMS- 0 and SGT-PMS- $\mathrm{x}(\mathrm{x}=1.5,2.2,3.0)$, respectively. The d-spacings of PMS-0, SGT-PMS-1.5, SGT-PMS-2.2, and SGT-PMS-3.0 were $11.35,10.82,11.95$, and $12.30 \mathrm{~nm}$, respectively. However, as shown in Fig. 4(a-d), the removal of the GO and P123 templates by calcination resulted in the decrease of d-spacing; $\mathrm{d}$-spacings of PMS-0, SGT-PMS-1.5, SGT-PMS-2.2, and SGT-PMS-3.0 were now $9.8,9.4,10.2$, and $10.8 \mathrm{~nm}$, respectively. This is attributed to the contraction of gallery owing to the removal of template. Figure 5 shows XPS spectra for the as-synthesized SGT-PMS-3.0 and SGT-PMS-3.0. The absence of carbon peak indicates that organic templates such as GO and P123 are removed completely by the calcination. Figure 6 shows SEM images of PMS-0 and SGT-PMS- $\mathrm{x}(\mathrm{x}=1.5,2.2,3.0)$. PMS-0 shows a regular particle spherical morphology [Fig. 6(a)]. However, SGT-PMS-x samples have particle morphologies different from that of PMS-0, exhibiting irregular and elliptical large particles with various sizes and shapes [Fig. 6(b-d)]. Here, small spherical particles [Fig. 6(b)], which are caused by the PMS-0 phase, disappeared on increasing the amount of GO added [Fig. 6(d)]. This is attributed to the decrease in the P123 micelles owing to the adsorption of P123 molecules on the GO surface in accordance with the increase in the amount of added GO.

The solid-state ${ }^{29} \mathrm{Si}$ MAS-NMR spectra of PMS-0 and SGT-PMS-3.0, as shown in Fig. 7(a,b), indicate that the pore wall structure of SGT-PMS-3.0 is fairly different from that of PMS-0. Here, SGT-PMS-3.0 is shown as 


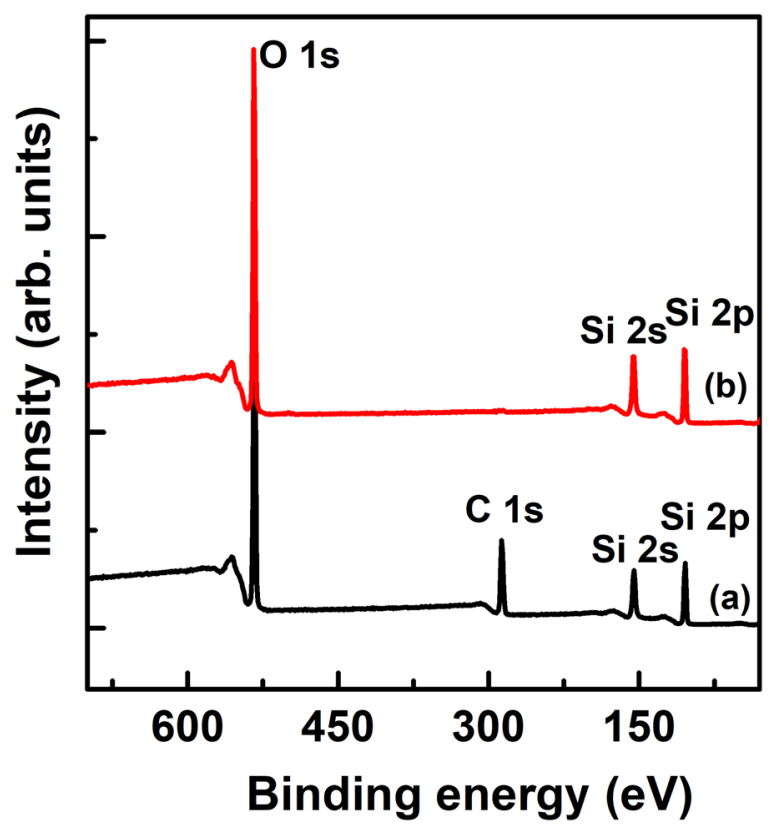

Figure 5. XPS spectra of (a) as-synthesized SGT-PMS-3.0, and (b) SGT-PMS-3.
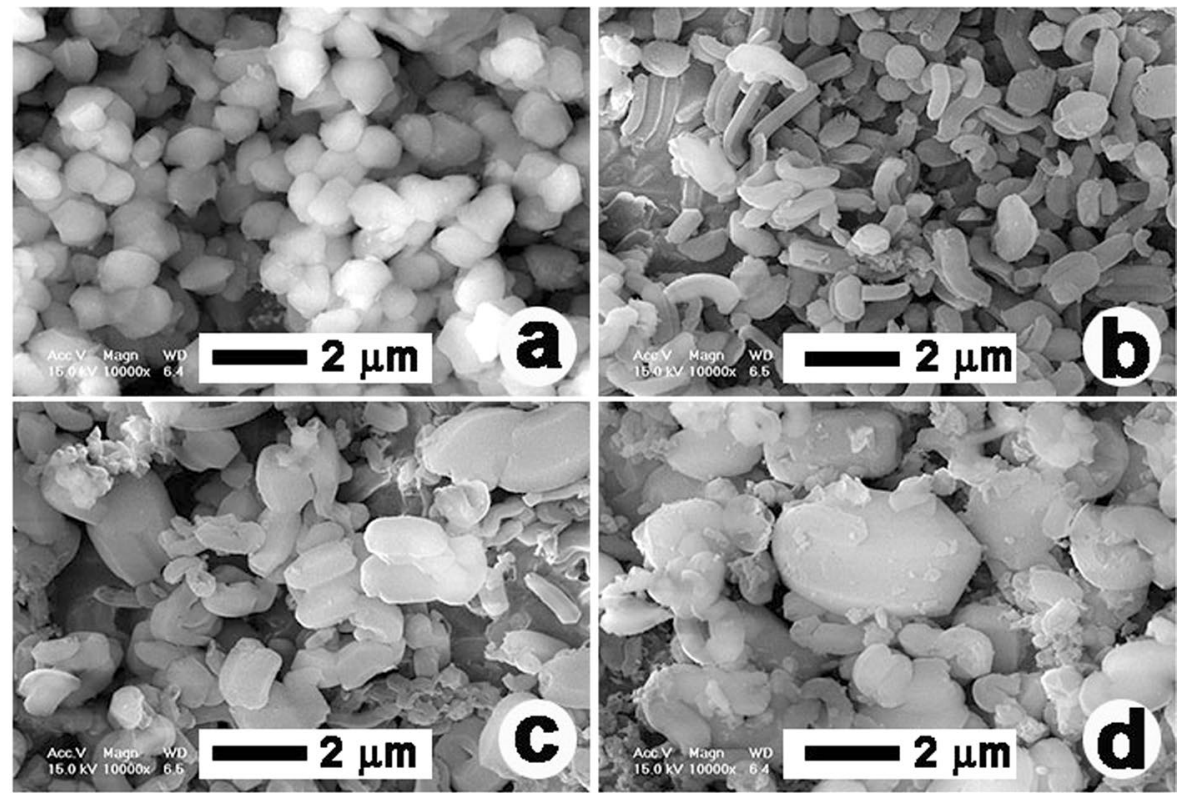

Figure 6. SEM images of (a) PMS-0, (b) SGT-PMS-1.5, (c) SGT-PMS-2.2, and (d) SGT-PMS-3.0.

a representative of SGT-PMS-x. In general, $\mathrm{Si}$ atoms of the silicate network exhibit signals $\left(\mathrm{Q}^{2}, \mathrm{Q}^{3}\right.$, and $\left.\mathrm{Q}^{4}\right)$ in the range of $\sim 90$ to $115 \mathrm{ppm}$. $\mathrm{Q}^{2}(\mathrm{HO})_{2} \mathrm{Si}(\mathrm{OSi})_{2}$ (near $\left.90 \mathrm{ppm}\right), \mathrm{Q}^{3} \mathrm{HOSi}(\mathrm{OSi})_{3}$ (near $\left.100 \mathrm{ppm}\right)$ and $\mathrm{Q}^{4} \mathrm{Si}(\mathrm{OSi})_{4}$ (110 to $1154 \mathrm{ppm}$ ) signals were observed due to the diverse environments of silicon. In particular, the intensity of $\mathrm{Q}^{3}$ and $\mathrm{Q}^{4}$ signals in the SGT-PMS-3.0 is more than double that of those for PMS- 0 . As the $\mathrm{Q}^{3}$ and $\mathrm{Q}^{4}$ signals are highly reflective of the degree of the silicate network development, this indicates that the pore walls in SGT-PMS-3.0 are well developed and firmer and thicker than those in PMS-0.

Figure 8 shows the Raman spectra of as-synthesized PMS-0 and SGT-PMS-x samples. Raman spectroscopy is employed to characterize the bonding, ordering, and crystallite size in carbon materials. A Raman band arises at $\sim 1582 \mathrm{~cm}^{-1}$ (the G-band) from the in-plane phonon modes of graphene, indicating $s p^{2}$ bonding. The D-band at $\sim 1353 \mathrm{~cm}^{-1}$ corresponds to disorder in the graphene layers caused by the presence of $s p^{3}$ bonding ${ }^{47}$. The peaks of GO observed at $\sim 1350$ and $\sim 1590 \mathrm{~cm}^{-1}$ correspond to the D and G bands, respectively ${ }^{48}$. However, in SGT-PMS- $x$, the D band occurs at $1315 \mathrm{~cm}^{-1}$, which is attributed to the influence of P123 adsorbed on GO surface. However, the G and D bands disappeared after calcination (Fig. 9), indicating that the GO and P123 


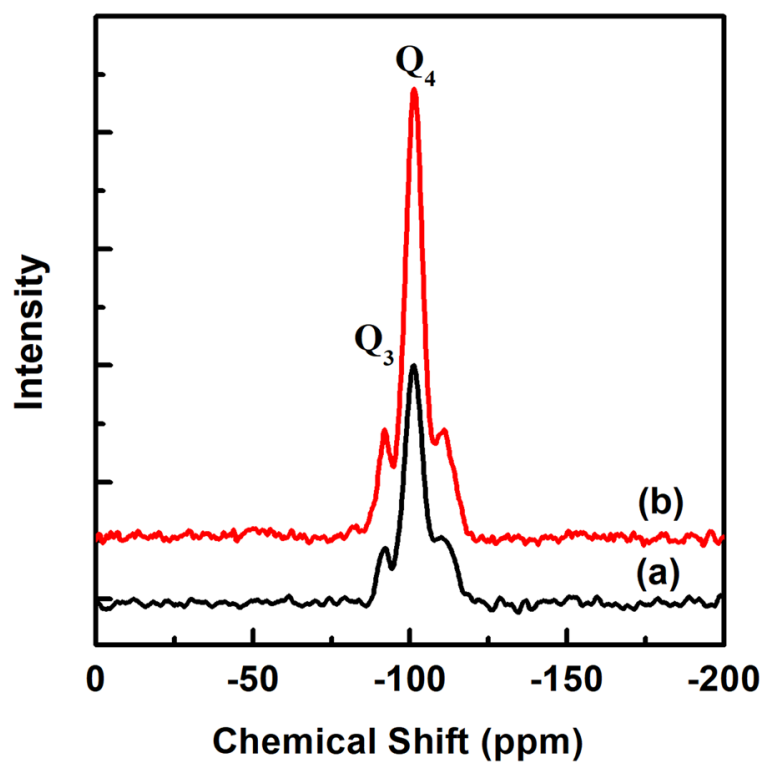

Figure 7. Solid-state ${ }^{29}$ Si MAS-NMR spectra of (a) PMS-0, and (d) SGT-PMS-3.0.

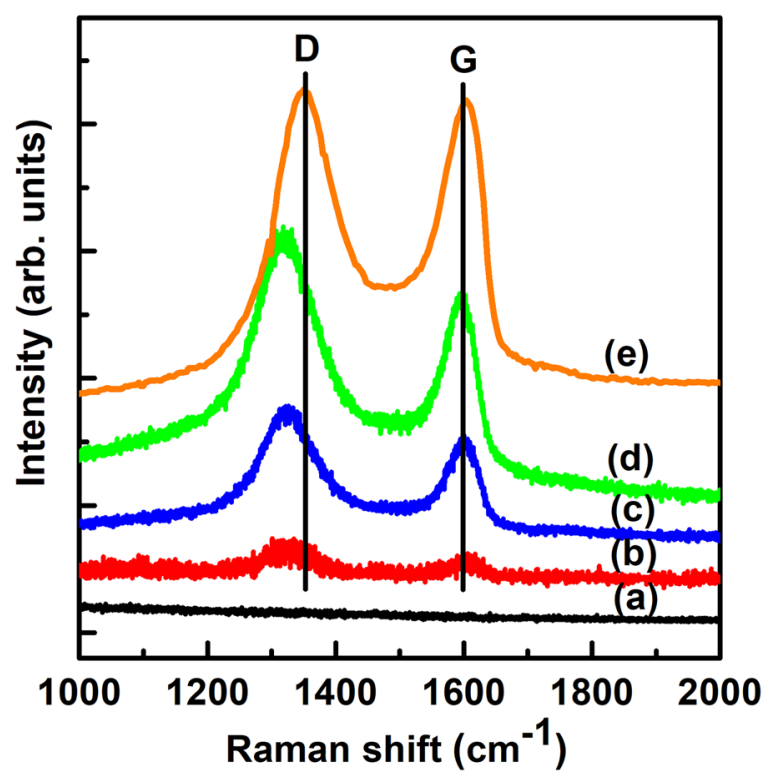

Figure 8. Raman spectra for as-synthesized samples of (a) PMS-0, (b) SGT-PMS-1.5, (c) SGT-PMS-2.2, and (d) SGT-PMS-3.0.

templates were removed during calcination. XPS peaks (Fig. 5) show that the GO and P123 templates are removed during calcination.

Figure 10 shows the $\mathrm{N}_{2}$ adsorption isotherms of PMS- 0 and SGT-PMS-x samples. A typical steep increase with mesopore filling at a relative pressure of $0.6-0.8$ is observed. The specific surface areas obtained using the Brunauer-Emmett-Teller (BET) equation are listed in Table 2, with considerably different values of 603.8, 713.6, 861.2, and $840.8 \mathrm{~m}^{2} \mathrm{~g}^{-1}$ for PMS-0, SGT-PMS-1.5, SGT-PMS-2.2, and SGT-PMS-3.0, respectively. The relatively high specific surface areas of PMS-0 and SGT-PMS-x samples indicate that PMS-0 and SGT-PMS- $x$ are silica microparticles with microporous and mesoporous structures. In particular, the increase in surface area with the addition of GO is attributed to the increase in micropores due to the development of a pore wall. In porous materials formed using several templates, specific surface area consists of micropores developed due to the pore walls and mesopores formed by burn-off of templates. Figure 11 shows the BJH pore-size distribution of PMS-0 and SGT-PMS-x samples; pore size of PMS-0, SGT-PMS-1.5, SGT-PMS-2.2 and SGT-PMS-3.0 is 5.8, 5.6, 5.3, and $5.1 \mathrm{~nm}$, respectively (Table 2). This is against the trend of $d$-spacing. In general, an increase in d-spacing results in an increase of pore size. However, if silicate sheets are thickened further by gelation using SGT, gallery height can be reduced relatively (because the gallery height $=d$-spacing - thickness of silica sheet), resulting in the decrease 


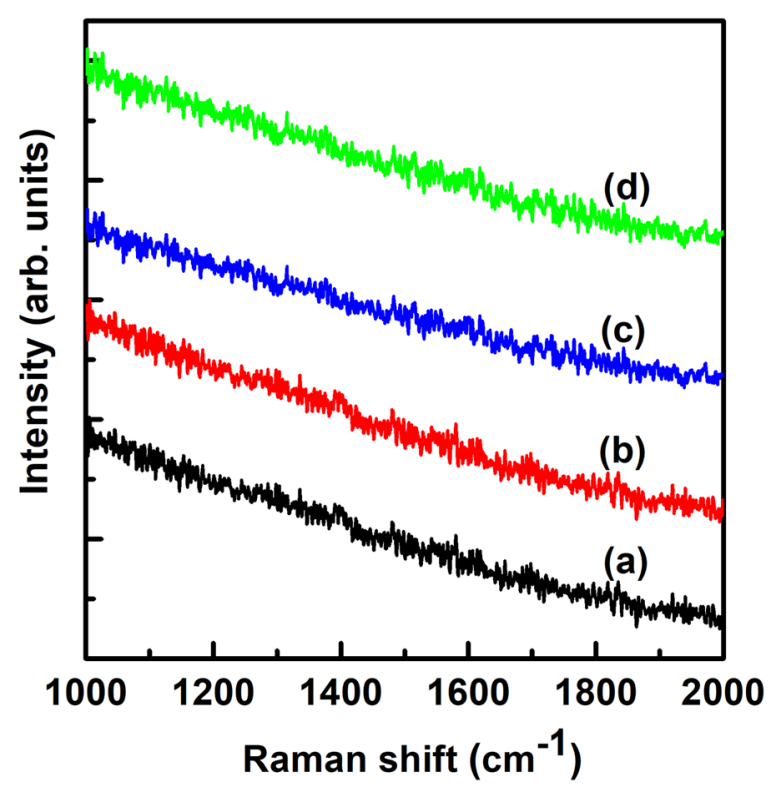

Figure 9. Raman spectra of (a) PMS-0, (b) SGT-PMS-1.5, (c) SGT-PMS-2.2, and (d) SGT-PMS-3.0.

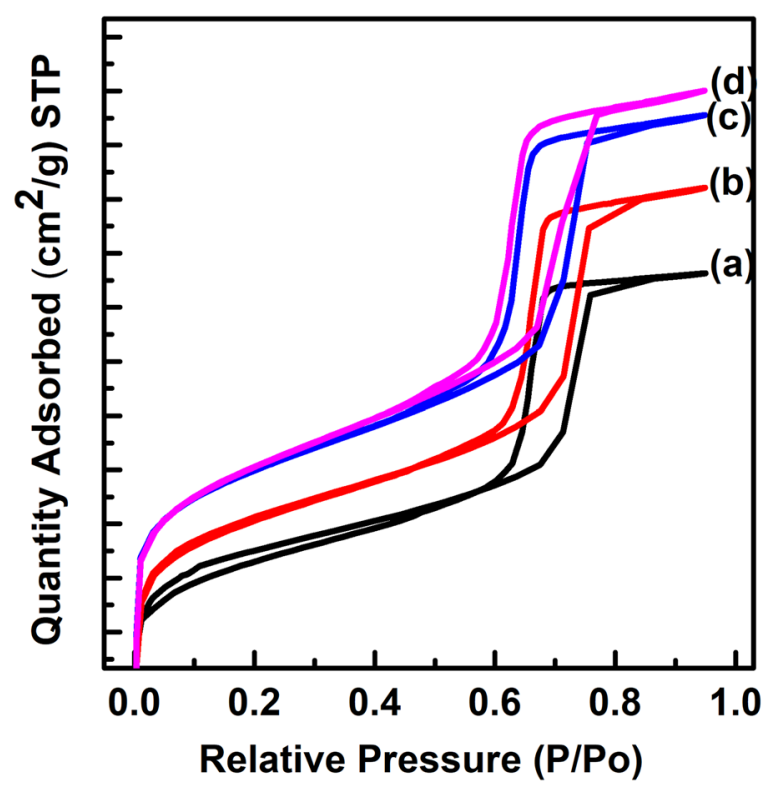

Figure 10. Nitrogen adsorption isotherms of (a) PMS-0, (b) SGT-PMS-1.5, (c) SGT-PMS-2.2, and (d) SGTPMS-3.0.

\begin{tabular}{|c|c|c|c|c|c|c|}
\hline \multirow{2}{*}{$\begin{array}{l}\text { Sample } \\
\text { PMS-0 }\end{array}$} & \multirow{2}{*}{$\frac{d_{100}(\mathbf{n m})}{9.82}$} & \multicolumn{3}{|c|}{\begin{tabular}{|l|} 
BET surface area $\left(\mathrm{m}^{2} \mathrm{~g}^{-1}\right)$ \\
$\mathrm{S}_{\text {total }} \mathrm{S}_{\text {mic }} \mathrm{S}_{\text {meso }}$ \\
\end{tabular}} & \multirow{2}{*}{$\begin{array}{l}\begin{array}{l}\text { Pore volume } \\
\left(\mathrm{cm}^{3} \mathrm{~g}^{-1}\right)\end{array} \\
0.8\end{array}$} & \multirow{2}{*}{\begin{tabular}{|l|}
$\begin{array}{l}\text { BJH pore diameter } \\
(\mathbf{n m}) \text { (adsorption) }\end{array}$ \\
5.8
\end{tabular}} \\
\hline & & 603.8 & 139.3 & \begin{tabular}{|l|l|}
464.3 \\
\end{tabular} & & \\
\hline SGT-PMS-1.5 & 9.36 & 713.5 & 190.4 & 523 & 0.85 & 5.6 \\
\hline SGT-PMS-2.2 & 10.24 & 840.8 & 196.5 & \begin{tabular}{|l|l|}
644.2 \\
\end{tabular} & 0.96 & 5.3 \\
\hline SGT-PMS-3.0 & 10.83 & 861.2 & 214.5 & 646.5 & 0.99 & 5.1 \\
\hline
\end{tabular}

Table 2. Physical properties of PMS-0 and SGT-PMS-x. Stotal $=$ BET surface area. $S_{\text {micro }}=$ Micropore volume from $\mathrm{t}$-plot; $\mathrm{t}=[14.3600 /(0.1013-\lg (\mathrm{P} / \mathrm{P} 0))]^{0.5}$. $\mathrm{S}_{\text {meso }}=\mathrm{S}_{\text {total }}-\mathrm{S}_{\text {micro }}$.

in pore size accompanied by the increase in surface area. This was already explained by the increase of the intensities of $\mathrm{Q}^{3}$ and $\mathrm{Q}^{4}$ peaks with the addition of GO. The increase of silica sheet thickness and the decrease of gallery height can bring out the increase of surface area, allowing the development of micropores. Therefore, the increase 


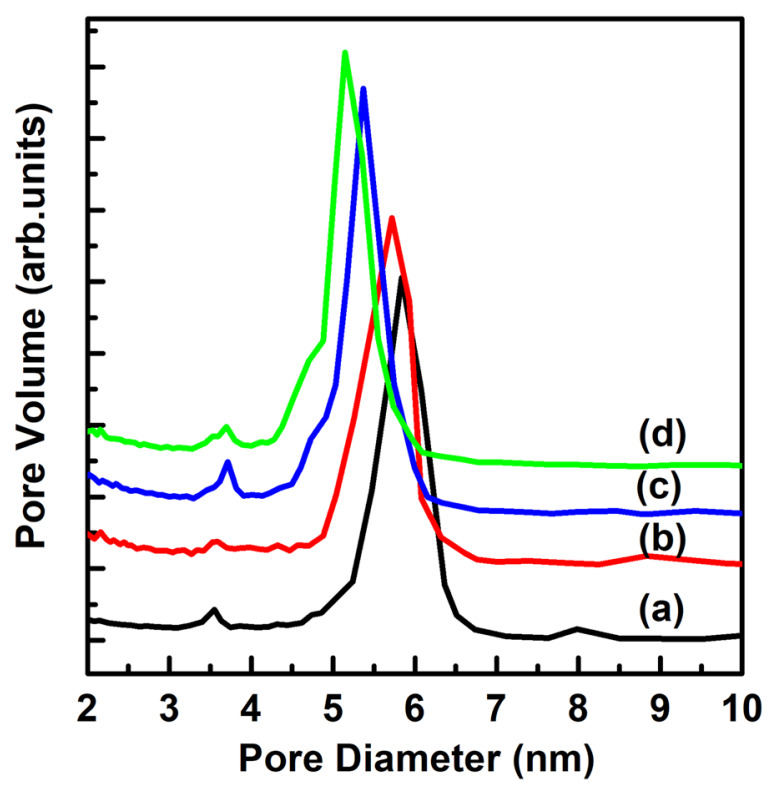

Figure 11. Pore size distribution obtained from the BJH adsorption isotherms of (a) PMS-0, (b) SGT-PMS-1.5, (c) SGT-PMS-2.2, and (d) SGT-PMS-3.0.
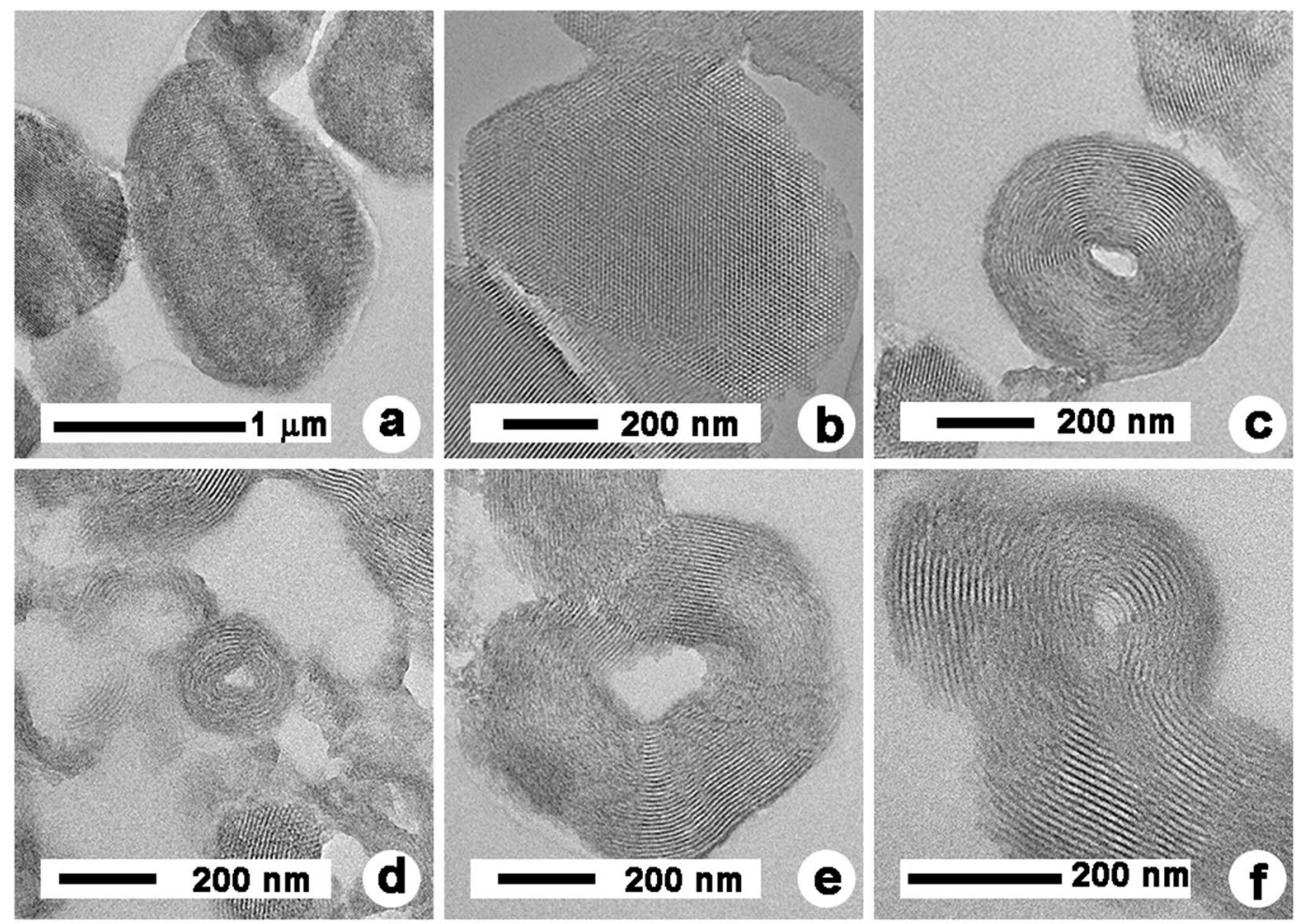

Figure 12. TEM images of (a,b) PMS-0, (c,d) SGT-PMS-2.2, and (e,f) SGT-PMS-3.0.

in surface area accompanied by the decrease of pore size are attributed to the increase in silica sheet thickness by the formation of SGT-PMS.

TEM images, as shown in Fig. 12, show the cross-section structures of SGT-PMS particles which cannot be confirmed by XRD peaks, SEM images, and ${ }^{29} \mathrm{Si}$ MAS-NMR spectra. These prove that large irregular particles, as shown in SEM, are rolled in silicate sheets stacked with regular spacing. The rolls with a layered structure are also accompanied by spherical particles with nanosized hexagonal pores. Here, the nanosized hexagonal pores originated from PMS-0, whereas the rolls with layered structure originated from SGT-PMS. These results indicate that SGT-PMS is a novel mesoporous silica phase formed by SGT. 


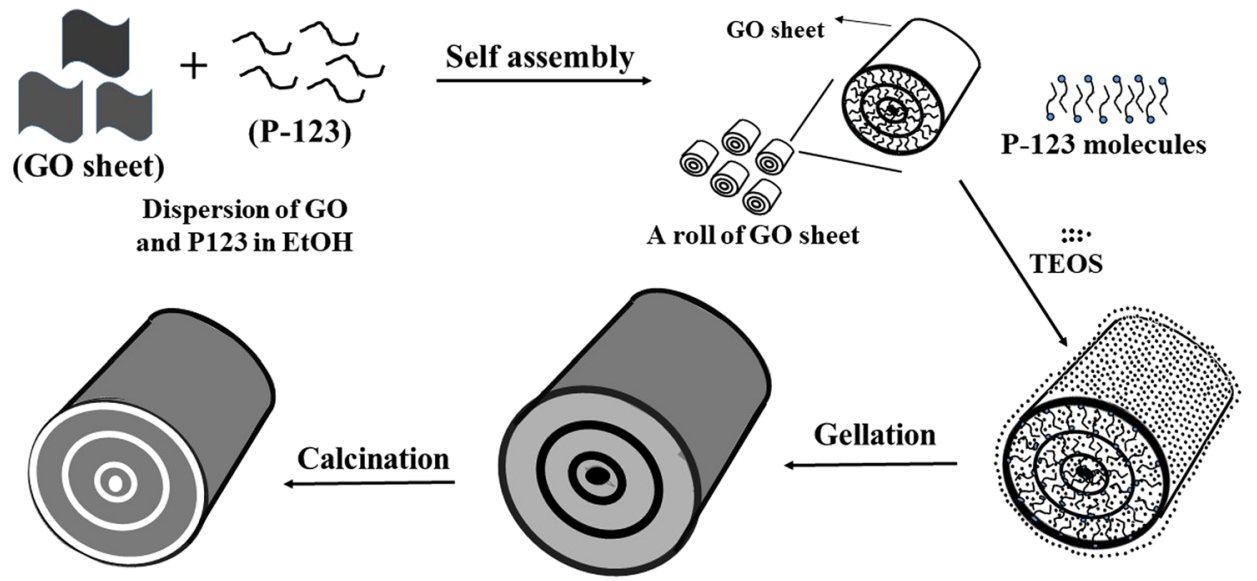

\section{Microporous \& mesoporous materials with gallery}

Figure 13. Schematic of SGT-PMS-x formed by self-assembled GO templating mechanism.

The schematic in Fig. 13 shows the SGT mechanism as a representation of the effect of the addition of GO on the physical properties such as particle morphology, regular pore structure, and surface area of SGT-PMS-x. When GO sheets are added to the P123 solution, hydrophilic head groups of P123 molecules are adsorbed on the surface of GO sheets by the molecular interaction between $\mathrm{OH}$ groups of P123 and $\pi$-electrons of GO sheet, resulting in hydrophobic GO sheets surrounded by alkyl chains. Such hydrophobic GO sheets form SGT like a roll stacked with GO sheets owing to neutral interaction between the alkyl chains. TEOS can be introduced into the hydrophobic galleries between GO sheets of SGT. The gelation of TEOS in the gallery of SGT results in two-dimensional thick silica sheets. The removal of the GO and P123 templates from as-synthesized samples leave only rolls comprising stacked silica sheets with regular spacing. Here, when GO is added in a small amount, SGT are accompanied by P123 micelle templates, resulting in a mixture of PMS-0 and SGT-PMS phases. The increase in the added amount of GO results in the increase in SGT-PMS-x particle size, thus leading to an increase in SGT size. Here, the galleries between silicate sheets are very regular because they are made of SGT supported by wide hydrophobic GO sheets. In future studies, we expect that mesoporous materials with various metal oxides can be produced by self-assembled GO templated gelation.

\section{Conclusion}

Novel mesoporous silicas were prepared successfully by the gelation of TEOS using a self-assembled GO template. SGT-PMS-X showed well-developed XRD peaks with $d$-spacings of 9.8-10.8 nm depending upon the amount of GO, thereby indicating mesoporous structures. The specific surface areas were increased from 603.8 to 861.2 $\mathrm{m}^{2} \mathrm{~g}^{-1}$, depending upon the amount of GO. The pore size distribution remained in the range $5.1-5.8 \mathrm{~nm}$ and the pore volume was in the range $0.80-0.99 \mathrm{~m}^{3} \mathrm{~g}^{-1}$. However, the pore size distribution remained in the range 5.1-5.8 nm. In particular, SEM images of SGT-PMS-x showed irregular and elliptical large particles with various sizes, which are fairly different from that of PMS-0. TEM images indicate that SGT-PMS particles comprises silicate sheets with regular spacing, which are fairly different from that of PMS-0 without GO. These results were well explained by the self-assembled GO templating mechanism. The above results indicate that mesoporous materials with various metal oxides can be produced by using self-assembled GO templates.

Received: 1 November 2019; Accepted: 23 March 2020;

Published online: 10 April 2020

\section{References}

1. Zhao, D. Y. et al. Triblock copolymer syntheses of mesoporous silica with periodic 50 to 300 angstrom pores. Science 279, 548-552 (1998).

2. Li, X. M. et al. Anisotropic growth-induced synthesis of dual-compartment Janus mesoporous silica nanoparticles for bimodal triggered drugs delivery. J. Am. Chem. Soc. 136, 15086-15092 (2014).

3. Han, L. et al. Synthesis and characterization of macroporous photonic structure that consists of azimuthally shifted doublediamond silica frameworks. Chem. Mater. 26, 7020-7028 (2014).

4. Parlett, C. M. A., Wilson, K. \& Lee, A. F. Hierarchical porous materials: catalytic applications. Chem. Soc. Rev. 42, 3876-3893 (2013).

5. Chen, Z. et al. DNA translocation through an array of kinked nanopores. Nat. Mater. 9, 667-675 (2010).

6. Dunphy, D. R., Sheth, P. H., Garcia, F. L. \& Brinker, C. J. Enlarged pore size in mesoporous silica films templated by Pluronic F127: use of poloxamer mixtures and increased template/ $\mathrm{SiO}_{2}$ ratios in materials synthesized by evaporation-induced self-assembly. Chem. Mater. 27, 75-84 (2015).

7. Wei, J., Yue, Q., Sun, Z. K., Deng, Y. H. \& Zhao, D. Y. Synthesis of dual-mesoporous silica using non-ionic diblock copolymer and cationic surfactant as co-Templates. Angew. Chem., Int. Ed. 51, 6149-6153 (2012).

8. Li, W., Yue, Q., Deng, Y. H. \& Zhao, D. Y. Ordered mesoporous materials based on interfacial assembly and engineering. Adv. Mater. 25, 5129-5152 (2013)

9. Li, W. \& Zhao, D. Y. An overview of the synthesis of ordered mesoporous materials. Chem. Commun. 49, 943-946 (2013). 
10. Huang, X. D., Zhou, L., Yu, C. Z. \& Zhao, D. Y. Self-assembly of monodispersed silica nano-spheres with a closed-pore mesostructure. J. Mater. Chem. 22, 11523-11528 (2012).

11. Corma, A. From microporous to mesoporous molecular sieve materials and their use in catalysis. Chem. Rev. 97, 2373-2420 (1997).

12. Kang, Y. et al. Uniform nanozeolite microspheres with large secondary pore architecture. Chem. Mater. 18, 1861-1866 (2006).

13. Kresge, C. T., Leonowicz, M. E., Roth, W. J., Vartuli, J. C. \& Beck, J. S. Ordered mesoporous molecular sieves synthesized by a liquidcrystal template mechanism. Nature 359, 710-712 (1992).

14. Beck, J. S. et al. A new family of mesoporous molecular sieves prepared with liquid crystal templates. J. Am. Chem. Soc. 114, 10834-10843 (1992).

15. Beck, J. S. et al. Molecular or supramolecular templating, defining the role of surfactant chemistry in the formation of microporous and mesoporous molecular sieves. Chem. Mater. 6, 1816-1821 (1994).

16. Jeong, S. Y., Suh, J. K., Lee, J. M. \& Kwon, O. Y. Preparation of silica-based mesoporous materials from fluorosilicon compounds: gelation of H2SiF6 in ammonia surfactant solution. J. Colloid Interface Sci. 192, 156-161 (1997).

17. Huo, Q., Leon, R., Petroff, P. M. \& Stucky, G. D. Mesostructure design with gemini surfactants, supercage formation in a threedimensional hexagonal array. Science 268, 1324-1327 (1995).

18. Tanev, P. T. \& Pinnavaia, T. J. A neutral templating route to mesoporous molecular sieves. Science 267, 865-867 (1995).

19. Ryoo, R., Kim, J. M., Ko, C. H. \& Shin, C. H. Disordered molecular sieve with branched mesoporous channel network. J. Phys. Chem. 100, 17718-17721 (1996).

20. Inagaki, S., Koiwai, A., Suzuki, N., Fukushima, Y. \& Kuroda, K. Syntheses of highly ordered mesoporous materials, fsm-16, derived from kanemite. Bull. Chem. Soc. Jpn. 69, 1449-1457 (1996).

21. Inagaki, S., Ogata, S., Goto, Y. \& Fukushima, Y. Mesoporous materials derived from layered silicates and the adsorption properties. Stud. Surf. Sci. Catal. 117, 65-76 (1998).

22. Joo, S. H. et al. Ordered nanoporous arrays of carbon supporting high dispersions of platinum nanoparticles. Nature 412, 169-172 (2001).

23. Kim, J. Y., Park, K. W. \& Kwon, O. Y. Preparation of mesoporous silica by the rapid gelation of Na2SiO3 and H2SiF6 in aqueous surfactant solution. Micrpor. Mesopor. Mat. 285, 137-141 (2019).

24. Bagshaw, S. A., Prouzet, E. \& Pinnavaia, T. J. Templating of mesoporous molecular sieves by nonionic polyethylene oxide surfactants. Science. 269, 1242-1244 (1995)

25. Sierra, L., Lopez, B., Gil, H. \& Guth, J. L. Synthesis of mesoporous silica from sodium silica solutions and a poly (ethylene oxide)based surfactant. Adv. Mater. 11, 307-311 (1999).

26. Kwon, O. Y., Kim, S. W. \& Choi, S. W. Synthesis of mesoporous molecular sieves: hydrolysis of H2SiF6 by a non-ionic polyethyleneoxide surfactant template. Micropor. Mesopor. Mat. 27, 255-259 (1999).

27. Venugopal, E., Aswal, V. K. \& Kumaraswamy, G. Nanoparticle size controls aggregation in lamellar nonionic surfactant mesophase. Langmuir. 29, 9643-9650 (2013).

28. Wang, L., Gong, C., Yuan, X. \& Wei, G. Controlling the self-assembly of biomolecules into functional nanomaterials through internal interactions and external stimulations: A Review. Nanomaterials 9, 285 (2019).

29. Hummers, W. Jr. \& Offeman, R. Preparation of graphitic oxide. J. Am. Chem. Soc. 80, 1339-1339 (1958).

30. Liu, Y. et al. Synthesis of mesoporous silica/reduced graphene oxide sandwich-like sheets with enlarged and "funneling" mesochannels. Chem. Mater. 27, 5577-5586 (2015).

31. Wang, Z.-M. et al. Sandwich-type nanocomposite of reduced graphene oxide and periodic mesoporous silica with vertically aligned mesochannels of tunable pore depth and size. Adv. Funct. Mater. 27, 1704066 (2017).

32. Wang, Z.-M., Wang, W., Coombs, N., Soheilnia, N. \& Geoffrey, A. O. Graphene oxide-periodic mesoporous silica sandwich nanocomposites with vertically oriented channels. ACS Nano. 4(12), 7437-7450 (2010).

33. Novoselov, K. S. et al. Electric field effect in atomically thin carbon films. Science 306, 666-669 (2004).

34. Dikin, D. A. et al. Preparation and characterization of graphene oxide paper. Nature 448, 457-460 (2007).

35. Sharma, R., Baik, J. H., Perera, C. J. \& Strano, M. S. Anomalously large reactivity of single graphene layers and edges toward electron transfer chemistries. Nano Lett. 10, 398-405 (2010).

36. Dreyer, D. R., Park, S., Bielawski, C. W. \& Ruoff, R. S. The chemistry of graphene oxide. Chem. Soc. Rev. 39, 228-240 (2010).

37. Compton, O. C. \& Nguyen, S. T. Graphene oxide, highly reduced graphene oxide, and graphene: versatile building blocks for carbonbased materials. Small 6, 711-723 (2010).

38. Allen, M. J., Tung, V. C. \& Kaner, R. B. Honeycomb carbon: a review of graphene. Chem. Rev. 110, 132-145 (2010).

39. Wang, Z.-M., Hoshinoo, K., Shishibori, K., Kanoh, H. \& Ooi, K. Surfactant-mediated synthesis of a novel nanoporous carbon-silica composite. Chem.Mater. 15, 2926-2935 (2003).

40. Chu, Y.-H. et al. Synthesis of nanoporous graphite-derived carbon-silica composites by a mechanochemical intercalation approach. Langmuir 21, 2545-2551 (2005).

41. Stankovich, S. et al. Graphene-based composite materials. Nature 442, 282-286 (2006).

42. Huang, X., Qi, X., Boey, F. \& Zhang, H. Graphene-based composites. Chem. Soc. Rev. 41, 666-686 (2012).

43. Park, K.-W. Carboxylated graphene oxide- $\mathrm{Mn}_{2} \mathrm{O}_{3}$ nanorod composites for their electrochemical characteristics. J. Mater. Chem. A. 2, 4292-4298 (2014).

44. Hou, D., Liu, Q., Cheng, H., Zhang, H. \& Wang, S. Green reduction of graphene oxide via lycium barbarum extract. J. Solid. State. Chem. 246, 351-356 (2017).

45. Li, D., Müller, M. B., Gilje, S., Kaner, R. B. \& Wallace, G. G. Processable aqueous dispersions of graphene nanosheets. Nat. Nanotech. 3, 101-105 (2008).

46. Zhang, Y. P., Li, H. B., Pan, L., Lu, T. \& Sun, Z. Capacitive behavior of graphene-zno composite film for supercapacitors. Electroanal. Chem. 634, 68-71 (2009).

47. Ferrari, A. C. et al. Raman spectrum of graphene and graphene layers. Phys. Rev. Lett. 97, 187401 (2006).

48. Kudin, K. N. et al. Raman spectra of graphite oxide and functionalized graphene sheets. Nano. Lett. 8, 36-41 (2008).

\section{Author contributions}

K.W.P. performed the experiments. AFM/FT-IR/SEM/TEM/XRD/BET/XPS/RAMAN/NMR/Figure. 13 and all figure drawing were by K.W.P. and O.Y.K. were involved in manuscript writing.

\section{Competing interests}

The authors declare no competing interests.

Additional information

Correspondence and requests for materials should be addressed to K.-W.P. or O.-Y.K

Reprints and permissions information is available at www.nature.com/reprints. 
Publisher's note Springer Nature remains neutral with regard to jurisdictional claims in published maps and institutional affiliations.

(c) (i) Open Access This article is licensed under a Creative Commons Attribution 4.0 International License, which permits use, sharing, adaptation, distribution and reproduction in any medium or format, as long as you give appropriate credit to the original author(s) and the source, provide a link to the Creative Commons license, and indicate if changes were made. The images or other third party material in this article are included in the article's Creative Commons license, unless indicated otherwise in a credit line to the material. If material is not included in the article's Creative Commons license and your intended use is not permitted by statutory regulation or exceeds the permitted use, you will need to obtain permission directly from the copyright holder. To view a copy of this license, visit http://creativecommons.org/licenses/by/4.0/.

(c) The Author(s) 2020 